\title{
Article
}

\section{Neonatal screening and selective sonographic imaging in the diagnosis of developmental dysplasia of the hip}

Choudry, Qas A. and Paton, Robin W.

Available at http://clok.uclan.ac.uk/23154/

Choudry, Qas A. and Paton, Robin W. (2018) Neonatal screening and selective sonographic imaging in the diagnosis of developmental dysplasia of the hip. The Bone \& Joint Journal, 100-B . pp. 806-810. ISSN 2049-4394

It is advisable to refer to the publisher's version if you intend to cite from the work. http://dx.doi.org/10.1302/0301-620X.100B6.BJ-2017-1389.R1

For more information about UCLan's research in this area go to http://www.uclan.ac.uk/researchgroups/ and search for < name of research Group>.

For information about Research generally at UCLan please go to http://www.uclan.ac.uk/research/

All outputs in CLoK are protected by Intellectual Property Rights law, including Copyright law. Copyright, IPR and Moral Rights for the works on this site are retained by the individual authors and/or other copyright owners. Terms and conditions for use of this material are defined in the policies page.

\section{CLoK}

Central Lancashire online Knowledge www.clok.uclan.ac.uk

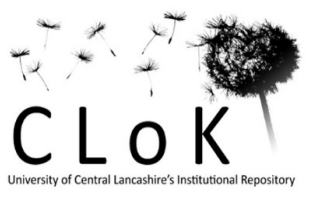




\section{The}

Bone \& Joint Journal

70 years of orthopaedic excellence

Neonatal clinical hip joint screening in combination with selective sonographic hip joint imaging in the diagnosis of developmental dysplasia of the hip (DDH).

\begin{tabular}{|r|l|}
\hline Journal: & The Bone \& Joint Journal \\
\hline Manuscript ID & BJJ-2017-1389.R1 \\
\hline Manuscript Type: & Original Article \\
\hline Keywords: & $\begin{array}{l}\text { Hip joint instability, Developmental Dysplasia of the Hip (DDH), } \\
\text { Screening,, Positive Predictive Value(PPV). }\end{array}$ \\
\hline \multicolumn{2}{|l}{} \\
\hline
\end{tabular}

SCHOLARONEm

Manuscripts 


\begin{abstract}
:
Aim: A prospective cohort study statistically evaluating the effectiveness of the neonatal hip instability, screening program.
\end{abstract}

Methods: A 4-year observational assessment of the neonatal clinical hip joint instability, screening program. All births underwent an Ortolani/Barlow manoeuvre within 72 hours of birth and positive cases were referred to the 'one stop' DDH, hip screening clinic (clinical and sonographic hip joint assessment). The results of this study were compared with previous published studies from this unit.

Results: There were 124 neonates referred as a positive Ortolani/Barlow manoeuvre, clunk positive or 'unstable'. There were only 5 cases of clinical hip instability confirmed in the 'one stop' clinic. Sonographically, there were 92 neonates with Graf Type I, 12 with Graf Type II and 20 Graf Type IV hips. Clinically, the Positive Predictive value (PPV) in the clinical neonatal hip screening programme was calculated as $4.0 \%$ and sonographically the PPV was $16.1 \%$.

Conclusion: Compared to previous published 10 year and 15 year studies from our unit, there has been a marked deterioration in the PPV in those referred as clinical hip instability. There appears to be a paradox of rising referrals but a decreasing PPV combined with increasing surgical intervention rates for DDH. 


\section{Introduction}

Developmental dysplasia of the hip is a spectrum of disorders ranging from mild hip dysplasia to irreducible hip dislocation [1]. In England, NIPE committee (Neonatal Infant Physical Examination) is responsible for screening guidelines. These consist of universal clinical hip instability screening within the first 72 hours post-natally, a General Practitioner / Health Care Professional clinical hip joint assessment at 6 weeks [2] and at 4 to 6 weeks a sonographic assessment of 'at risk' cases (breech presentation and strong family history of pathological hip dysplasia and dislocation). The Ortolani and Barlow manoeuvres are the internationally accepted techniques to identify clinical neonatal hip instability $[3,4]$. Despite the high specificity of both tests, traditionally the sensitivity of this clinical screening has been calculated as $60 \%[5]$. Previously published literature from our unit suggested that the quality of the hip screening had remained reasonably static over 10 to 15 years $[6,7]$. Duppe et al, in Sweden demonstrated deterioration in the results of their clinical hip screening programme which was attributed to increasing numbers of practitioners undertaking the clinical hip screening manoeuvres [8]. Anecdotally, it was felt that there had been a recent deterioration in the quality of referrals to our specialist DDH screening clinic. The aim of this study is to assess the positive predictive value of the initial screening clinical hip examination (as defined by positive Barlow/ortolani manouevre, clunk, hip instability) by non-expert compared with an expert in hip screening, either finding a positive provocation test or Graf Type IV ultrasound scan at approximately 2 weeks.

Previous studies have investigated the association of certain clinical signs with pathological DDH (asymmetrical skin creases, limited hip abduction and clicky hips) and their association with pathological DDH. These associations were not investigated in this study $[9,10,11]$. 


\section{Patients \& Methods}

A prospective longitudinal observational study performed at the Royal Blackburn Teaching Hospital from $1^{\text {st }}$ January 2012 to $31^{\text {st }}$. December 2015 inclusive. The current birth rate is slightly over 7,000 live births per year with a current total population of 530,000 in the 5 districts covered (children and adults). Since 1992, all cases of neonatal instability referred to the 'one stop' DDH hip joint screening clinic have been clinically assessed and the hip joints sonographically imaged by the senior author. The information is prospectively recorded on individual data sheets that is transferred to a spreadsheet and is adapted into a database. All cases of neonatal hip instability identified in the district were referred directly to this clinic by the Paediatric department or by midwives using an agreed proforma. The clinical neonatal hip joint examination was undertaken by differing health professionals ranging from newly qualified medical doctors [foundation doctors, doctors not in training, midwives and Advanced Nurse Practitioners (ANPs)]. All had received training in neonatal hip joint examination.

Inclusion criteria included; a positive provocative test (Ortolani and/or Barlow), patients referred with a positive 'clunk' on the Ortolani manoeuvre and hip joints referred as positive for 'instability'( Figure 1). 
Figure 1: Flow diagram for the assessment \& referral of potential neonatal hip joint instability (DDH screening)

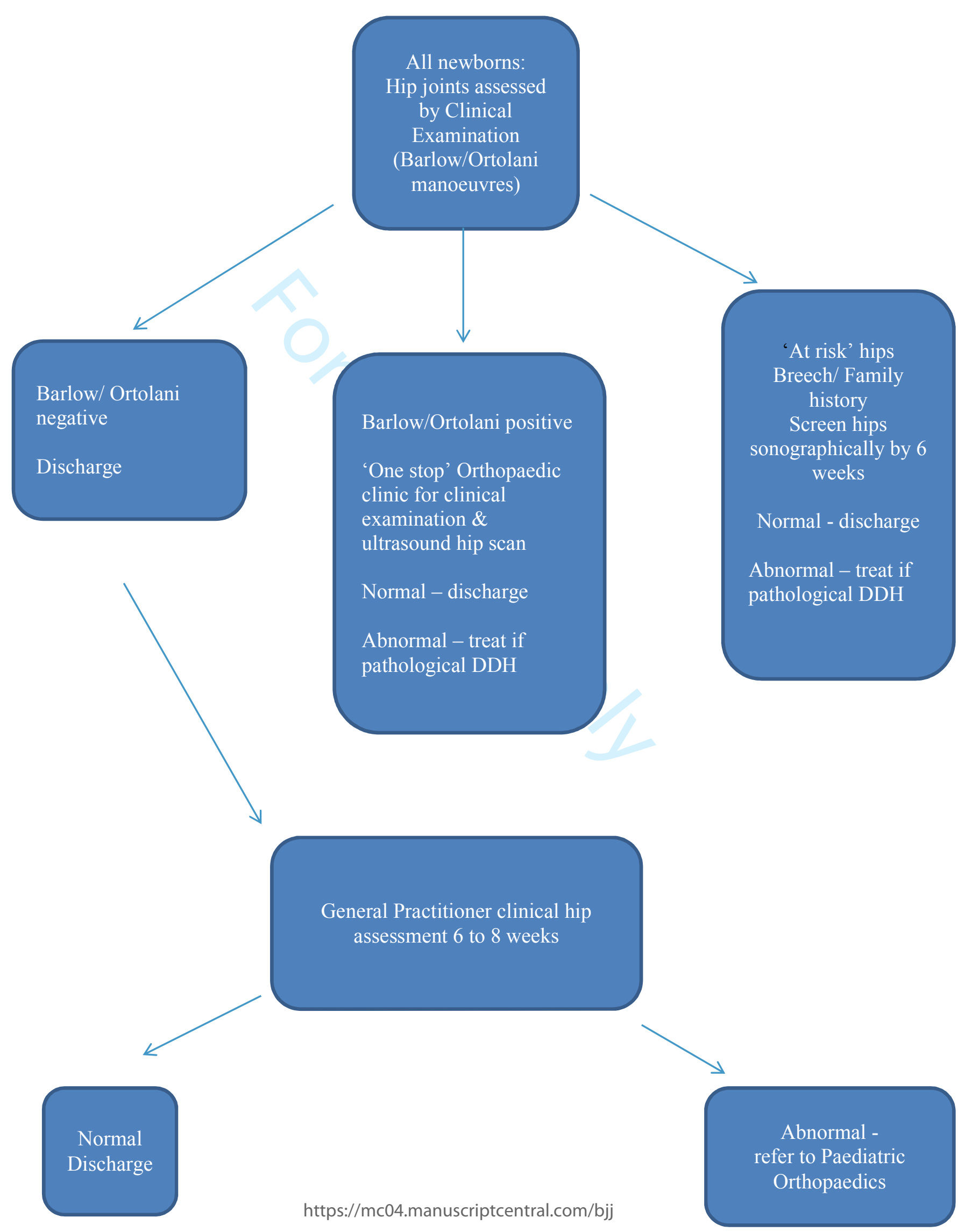


Exclusion criteria included referrals to the clinic for reasons other than potential neonatal hip joint instability i.e. 'at risk' factors, clicky hips, asymmetrical skin creases, limitation of hip joint abduction (bilateral and unilateral) and primary neurological or syndromic hip pathology. The diagnosis of DDH does not include neurological or syndromic causes, as these cases are secondary to a primary pathology [12]. Patients not referred through the neonatal hip joint screening programme were deemed 'late' presentations and were excluded from the primary analysis of neonatal instability, though 'late' presentation cases of irreducible hip dislocation requiring surgical intervention were recorded in order to calculate the sensitivity of the study.

In the 'one stop' clinic the hip joints were clinically assessed using the Ortolani and Barlow manoeuvres. Sonographic imaging of the hip was classified according to modified Graf and Harcke classification (Table 1) [13,14,15]. 
Table 1: Modified Graf and Harcke sonographic classification of the hip joint.

\begin{tabular}{|l|l|l|}
\hline & Alpha angle & $\begin{array}{l}\text { Hip joint (position of } \\
\text { the femoral head in the } \\
\text { acetabulum) }\end{array}$ \\
\hline Graf Type I & $>60$ degrees & Congruous \\
\hline Graf Type II & $43-60$ degrees & Congruous \\
\hline Graf Type III & $<43$ degrees & Congruous \\
\hline Graf Type IV & Any degree & Subluxated or dislocated \\
\hline
\end{tabular}

The outcome measures of this study were an unstable hip [a positive provocative test in clinic (Ortolani or Barlow manoeuvre) or a sonographic Graf Type IV hip joint]. It is accepted in some quarters that a positive sonographic Graf Type IV scan may over diagnose the condition when compared to a clinical Ortolani/ Barlow positive manoeuvre and may be a flawed outcome measure [6].

Sensitivity, specificity and Positive Predictive Value (PPV) were calculated for both the clinical and the sonographic assessment. In the sonographic group an assumption was made that all referred clinically unstable hip joints would be expected to have 'on the balance of probabilities' a Graf Type IV sonographic image on primary hip examination. Without this assumption the sonographic PPV would not be able to be calculated. Birth rates for the districts covered by the East Lancashire Hospital NHS Trust were obtained from the Office for National Statistics. 
A separate prospective spreadsheet based was maintained for the number of cases of irreducible hip dislocation, subluxation and hip dysplasia cases that required surgical intervention (Closed reduction, open reduction, femoral osteotomy and pelvic osteotomy). For the purposes of statistical analysis for this study, a false negative result was an irreducible hip dislocation that presented 'late' after the neonatal screening period (outcome measure). Data was collected prospectively on and for at least 18 months after the end of the study in order to identify all cases of irreducible hip dislocation born within the 4-year study period.

A separate card index system was maintained prospectively on all cases diagnosed with sonographic hip abnormalities and or Pavlik harness treatment (within the 4year study period). This was a cross checking system, separate to the primary database, in order to identify and separate, early from late diagnosed pathological cases.

\section{Results}

Between $1^{\text {st }}$. January 2012 and $31^{\text {st }}$ December 2015, 124 patients were referred through the 'one stop clinic' with clinical hip instability: 100 as positive Ortolani/ Barlow manoeuvres, 15 unstable and 9 clunks. There were 28,241 live births.

The mean age at assessment in the 'one stop' clinic' was 16.1 days (95\% $\mathrm{Cl}+/-2.1)$ in those who did not fail to attend (FTA) their first appointment. These neonates were seen and clinically assessed by the treating expert within 4 weeks of the referral (NIPE guideline). 
Ten cases FTA their initial appointment and attended at a later date. Mean age at assessment of these cases was 73.5 days $(95 \% \mathrm{Cl}+/-17.5)$. All these patients had normal clinical examinations with the following ultrasound findings. Nine Graf Type I hips and 1 Graf Type II hip. All hip abnormalities resolved and did not require treatment.

On clinical assessment in this 'one stop' clinic by the senior author, only 5 patients over the 5 years demonstrated a positive provocative test.

On sonographic assessment, 92 patients (74.1\%) had a Graf Type I hip joint and a normal hip joint on clinical examination. These patients were discharged from the clinic. Eighteen neonates presented initially with a Graf Type II hip joint with a normal clinical hip joint examination. Twelve of the 18 Graf Type II hips resolved to normal Graf Type I hip joints spontaneously, with 6 deteriorating and progressing to Graf Type IV hip joints. Fourteen Graf Type IV hip joints were diagnosed at the initial clinic appointment (20 Graf Type IV including the 6 Graf Type II hips that progressed to Graf Type IV). Graf Type IV hips were treated by Pavlik harness and the majority resolved, although 7 hip joints progressed and required later surgical intervention (1 closed reduction and 6 open reductions of the hip joints).

From 2012 to 2015 the rate of surgical intervention for irreducible hip dislocation and dysplasia in DDH was 1.1 per 1000 live births compared to 0.62 per 1000 from 19972006 [6]. The rate of surgery for irreducible dislocation increased to 0.96 per 1000 compared to 0.51 per 1000 over the same time periods [6]. 
Compared to the previous 15 years, the mean yearly referral number of clinical hip instability, increased from 13.4 to 31 (3.18 referrals per 1000 live births has increased to 4.4 per 1000 live births).

The PPV for clinical assessment and sonographic assessment was calculated. The PPV for clinical Ortolani/ Barlow positive in the 'one stop hip clinic' was 4.0\% (5/124). If referrals for 'instability' are excluded and clunks and Ortolani/Barlow positive are calculated the PPV would still be only 5.0\% (5/100). The PPV for sonographic assessment (proportion of patients referred with instability that were found to have a Graf Type IV hip on imaging) was $16.1 \%$ (20/124). The sensitivity of the clinical assessment was $18.5 \%$ and in the sonographic assessment was $47.6 \%$. The specificity of the clinical and sonographic assessment was $99.6 \%$. The results are summarised in Tables 2 and 3.

Table 2: Results of clinical 3examination and sonographic imaging in the 'one stop' clinic

\begin{tabular}{|c|c|c|}
\hline & Definition & Number \\
\hline True negatives & Normal hips which were not referred & $\begin{array}{l}\text { Clinical }=28,095 \\
\text { Sonographic }=28,095\end{array}$ \\
\hline \multirow[t]{2}{*}{ True positives } & \multirow{2}{*}{$\begin{array}{l}\text { Referred as unstable hips and were } \\
\text { diagnosed as unstable in the 'one } \\
\text { stop' clinic }\end{array}$} & Clinical $=5$ \\
\hline & & Sonographic $=20$ \\
\hline False negatives & $\begin{array}{l}\text { Irreducible hip joint dislocation not } \\
\text { referred in the neo-natal period; } \\
\text { "late dislocations" }\end{array}$ & 22 \\
\hline \multirow[t]{2}{*}{ False positives } & \multirow{2}{*}{$\begin{array}{l}\text { Referred as unstable hips but were } \\
\text { diagnosed as stable in the 'one stop' } \\
\text { clinic }\end{array}$} & Clinical $=119$ \\
\hline & & Sonographic $=104$ \\
\hline
\end{tabular}


Table 3: Comparison of 3 sonographic and clinical screening studies.

\begin{tabular}{|l|l|l|l|}
\hline & Current Study & Mace et al 2015 & Paton 2011 \\
\hline Time period of study & $\begin{array}{l}2012-2015 \\
(4 \text { years })\end{array}$ & $\begin{array}{l}1997-2011 \\
(15 \text { years) }\end{array}$ & $\begin{array}{l}1997-2006 \\
(10 \text { years })\end{array}$ \\
\hline Sensitivity (sonographic) & $47.6 \%$ & $77 \%$ & $72 \%$ \\
\hline Sensitivity (clinical) & $18.5 \%$ & $62 \%$ & $66 \%$ \\
\hline Specificity (sonographic) & $99.6 \%$ & $99.8 \%$ & $99.9 \%$ \\
\hline Specificity (clinical) & $99.6 \%$ & $99.8 \%$ & $99.8 \%$ \\
\hline PPV (sonographic) & $16.1 \%$ & $47 \%$ & $68 \%$ \\
\hline PPV (clinical) & $4.0 \%$ & $24 \%$ & $28 \%$ \\
\hline
\end{tabular}

\section{Discussion}

Screening for the early detection of pathological DDH is a controversial subject $[16,17,18]$. There is no international consensus $[2,19,20,21]$.

Evidence is lacking that this NIPE screening programme has resulted in a true reduction in late presenting dislocation rates in pathological DDH in England [6,22, $23,24,25]$. In the UK, despite a hip screening programme instituted in 1969, over 60 percent of irreducible hip dislocations present late, often after the age of 1 year [22]. The overall rate of surgery irreducible hip dislocation prior to selective sonographic 
hip joint screening in the UK was between 0.5 and 0.8 per 1000 live births $[23,24,25]$. However, Duppe et al in Sweden and Myers et al in New Zealand have shown a significant reduction in the numbers and rates of surgery for pathological DDH when a small group of well trained, experienced hip joint examiners undertake the primary clinical neonatal hip joint screening $[8,26]$. A recent study from Australia has recorded a concerning increase in irreducible hip dislocation rates [27].

In the UK, concern has been expressed on those who are currently responsible for undertaking the neonatal hip joint examination. They have varying degrees of training and clinical experience. Recently, there has been a switch to stand alone 'birthing centres', resulting in more births out with the traditional hospital maternity unit. It is not clear if these changes may have affected the effectiveness of the screening programme [28].

The natural history of resolution of neonatal hip instability has been well documented in the literature. Gardiner \& Dunn and Barlow have reported that $71 \%$ of clinically unstable hips stabilise within 2 weeks and $88 \%$ stabilise by the first month postnatally without treatment $[4,29]$. This may be a limitation to the objective nature of this study. However, as most of the hip joints in this study were assessed in the 'one stop' clinic close to 2 weeks post-natally it would be expected that the clinical PPV would be between 20 to $30 \%$ and the sensitivity to be between 60 to $70 \%$, based on published studies $[5,6,7]$.

Our previous published data had shown that the PPV and sensitivities for neonatal hip instability screening remained reasonably static over 10 to 15 years $[6,7]$ Table 3 . 
The PPV in this current clinical neonatal hip examination programme, has fallen to $4.0 \%$ compared to 24 to $28 \%$ in previous studies. The PPV of sonographic Graf Type IV imaging, has fallen to $16.1 \%$ compared to $49 \%$ and $68 \%$ in the previous studies $[6,7]$. Although the referral criteria of hip instability and the pathways in the previous 2 studies are the same, unidentified subtle differences and confounding factors in data collection could make direct comparison with this study less robust than expected. The clinical hip examinations and sonographic hip imaging was undertaken by the same examiner. This increases the likely hood of unintentional bias though this was unavoidable in this study as the clinic was consultant based only. The senior author however has over 20 years of sonographic hip imaging experience and his ultrasonography image quality and interpretation have been independently validated as accurate at a national level (NIPE committee, a subgroup of the National Screening Committee).

Neonatal clinical hip screening in the UK appears fragmented with numerous stakeholders. Guidance in England is the responsibility of Public Health England [30]. Local health commissioning is the responsibility of the Clinical Commissioning Groups (CCG) and the hospital neonatal clinical hip examination/ screening is the responsibility of the Paediatric department. In the community ('birthing centres'), midwives and nurses undertake the neonatal hip screening. Due to the low incidence of true clinical hip instability (Ortolani/Barlow positive) many undertaking the clinical hip screening will have little experience of exposure to true hip joint instability (positive provocative manoeuvres). It is of concern, that this current study identified an apparent increase in the operative rate for irreducible hip dislocation DDH of 0.96 per 1000 live births over a 4-year period, compared to 0.51 per 1000 live births in the 
same institution in a 10-year period [6]. An additional possible driver of the increase in referrals and of false positives may be the fear of litigation.

There is continuing controversy on if universal or selective sonographic hip screening is cost effective $[12,13,14,15,16,17]$. Woodacre et al [31] calculated the cost of closed or open reduction hip joint procedures varied from $£ 4,352$ to $£ 7.052$ per case. This is compared with approximately $£ 41$ for a hip ultrasound scan plus and additional $£ 156$ for a 'one stop clinic with hip joint sonographic imaging performed by a Consultant Orthopaedic Surgeon [9]. If universal ultrasound scanning is undertaken in all neonates, by the radiological department alone, the cost in our service would be approximately $£ 290,000$ per year.

The medical negligence cost of delayed diagnosis that later requires surgery ranges from $£ 120,000$ to $£ 488,000$ (out of court settlements)[32,33].

However the early detection of pathological DDH does not prevent all surgical intervention as some cases do not respond to treatment in the Pavlik harness [7,34 ].

\section{Conclusion}

The results of our study mirror the Swedish experience [8]. There appears to be a paradox of increasing referrals of neonatal hip instability combined with a decreasing PPV and an increase in surgical intervention for DDH. The large number of examiners of different backgrounds and experience undertaking neonatal hip screening for instability may be associated with an increase in false positive and false negative referrals. It is important to limit hip screening for $\mathrm{DDH}$ to a small group of trained and experienced individuals, for maximum effectiveness, if the resources allow. No National audit has been undertaken since the changes in the NIPE 
guidelines for neonatal hip screening were enacted in 2004. Is it not time to undertake a National audit to compare the current incidence of irreducible hip dislocation and pathological hip dysplasia to the results of the MRC study of 1998 in order to evaluate if the current NIPE screening policy in England is effective or not? [22]

\section{References:}

1. Klisic PJ, Congenital dislocation of the hip - a misleading term: brief report. $\mathrm{J}$ Bone Joint Surg. 71-B (1): 136, 1989

2. NHS Newborn \& Infant Physical Examination programme (NIPE) newbornphysical.screening.nhs.uk

3. Ortolani M, Un segno poco noto e sua importanza per la diagnosi precoce di prelussazione congenital dell'anca, la Pediatria 145, 129-36, 1937

4. Barlow TG, Early diagnosis and treatment of congenital dislocation of the hip. J Bone Joint Surg, 44-B (2), 292-301, 1962

5. Jones D, An assessment of the value of examination of the hip in the newborn. J Bone Joint Surg 59-B, 318-22, 1977

6. Paton RW, Does selective ultrasound imaging of 'at risk' hips and clinically unstable hips in Developmental Dysplasia of the hip (DDH)produce an effective screening programme. PhD Thesis, University of Lancaster uk.bl.ethos.618306, 2011

7. Mace J, Paton RW, Neonatal clinical screening of the hip in the diagnosis of developmental dysplasia of the hip. A 15 year prospective longitudinal study. Bone Joint J. 97-B (2), 265-9, 2015 
8. Duppe H, Danielsson LG, Screening of neonatal instability and of developmental dislocation of the hip. A survey of 132,601 living newborn infants between 1956 and 1999, J Bone Joint Surg 84-B (6), 878-85, 2002

9. Nie K, Rymaruk S, Paton RW, Clicky hip alone is not a true risk factor for developmental dysplasia of the hip. BJJ, 99-B (11): 1533-1536, 2017

10. Choudry Q, Goyal R, Paton RW, Is limitation of hip abduction a useful clinical sign in the diagnosis of developmental dysplasia of the hip. Arch Dis Child 98(11): 862-6, 2013

11. Anderton M, Paton RW, Isoloated asymmetrical skin creases and their association with pathological developmental dysplasia of the hip: A 21 year observational longitudinal study. BJJ 99-B (Supp 11): 15, 2017

12. Bialik V, Bialik GM, Blazer S et al, Developmental dysplasia of the hip: a new approach to incidence. Pediatrics 103 (1): 93-9, 1999

13. Graf R, Scott S, Farkas P et al, Manual for hip sonography. Edition Stolzalpe, 1999

14. Harcke HT, Clarke NM, Lee MS, Borns PF, MacEwan GD, Examination of the infant hip with real time ultrasonograhy. J Ultrasound Med, 3(3): 131-7, 1984

15. Rosendahl K, Markestad T, Lie RT, Ultrasound in the early diagnosis of congenital dislocation of the hip: significance of hip stability versus acetabular morphology Pediatr. Radiol. 22(6): 430-3, 1992

16. AAOS, Detection and non-operative management of Pediatric developmental dislocation of the hip in infants up to six months of age, AAOS.org, 2014

17. Cochrane review: Shorter D, Hong T, Osborn DA, Screening programmes for developmental dysplasia of the hip in newborn infants. Evid. Based Child Health 8(1): 11-54, 2013

18. Shipman SA, Helfand $M$, Meyer $V$ et al, Screening for developmental dysplasia of the hip: a systemic literature review for the US preventative services task force. Pediatrics 117(3): e557-76, 2006 
19. Rosendahl K, Markestad T, Lie RT, Ultrasound screening of Developmental Dysplasia of the Hip in the neonate: the effect on treatment rate and prevalence of late cases, Pediatrics 94(1): 47-52, 1994

20. Holen KJ, Tegnander A, Bredland T, et al, Universal or selective screening of the neonatal hip using ultrasound? A prospective, randomised trial of 15,529 newborn infants. J Bone Joint Surg 84-B(6): 886-90, 2002

21. Jones D, Dezateux CA, Danielsson LG et al, Topic for debate: At the crossroads - neonatal detection of developmental Dysplasia of the Hip. J Bone Joint Surg. 82-B(2): 160-4, 2000

22. Robinson R, Effective screening in child health, BMJ 316(7124): 1-2, 1998

23. Barnard AA, O'Hara JN, Bazin S et al, An improved screening system for the early detection of congenital dislocation of the hip. J Pediatr. Orthop 7(3): 27782,1987

24. Macnicol MF. Results of a 25-year screening programme for neonatal hip instability. J Bone Joint Surg Br. 1990; 72:1057-1060.

25. Godward S, Dezateux C, Surgery for congenital dislocation of the hip in the UK as a measure of outcome of screening. MRC Working Party on congenital dislocation of the hip. Medical Research Council. Lancet 351 (9110): 1149-52, 1998

26. Myers J, Hadlow S, Lynskey T, The effectiveness of a programme for neonatal hip screening over a period of 40 years: a follow up of the New Plymouth experience. J Bone joint Surg. 91-B (2), 245-8, 2009

27. Struder K, Williams N, Antoniou G et al, Increase in late diagnosed developmental dysplasia of the hip in South Australia: risk factors, proposed solutions. Med J Aust 204 (6): 240, 2016

28. Sewell MD, Rosendahl K, Eastwood DM. Developmental dysplasia of the hip. BMJ; 339:b4454, 2009 
29. Gardiner HM, Dunn PM, Controlled trial of immediate splinting versus ultrasonographic surveillance in congenitally dislocatable hips. Lancet 336 (8730): 1553-6, 1990

30. Newborn and infant physical examination (NIPE) screening: programme handbook 2016/17. PHE publications gateway number: 2015772, 2016

31. Woodacre T. Dhadwal A, Ball T, Edwards C, Cox PJA, The cost of late detection of developmental dysplasia of the hip, J Child Orthop 8(4): 325-332, 2015

32. IrwinMitchell Solicitors.

https://www.irwinmitchell.com/newsandmedia/2014/november/high-courtjudge-approves-settlement-from-nhs-trust-that-delayed-diagnosing-babysdislocated-hip-jq-713746

33. BoyesTurner, Medical Negligence Solicitors. https://www.boyesturnerclaims.com/site/news/birth-injury-news/undetectedhip-dysplasia-488k-compensation.

34. Choudry Q, Paton RW, Pavlik harness treatment for pathological developmental dysplasia of the hip: Meeting the standard. J Ped Orthop B 26(4): 293-7, 2017 